\title{
Evolución histórica y futura de la producción de setas en los pinares de Cataluña en función de distintos escenarios y modelos de cambio climático
}

Historical and future evolution of mushrooms productivity according different climate change scenarios and models in Catalonia pine forests

Morera, A. ${ }^{*}$ 


\title{
Resumen
}

Para este estudio, se determinaron los cambios en la producción de hongos con respecto a diferentes modelos y escenarios de cambio climático y registros históricos en Cataluña. Estos datos permitieron estimar y predecir la evolución histórica y futura de la producción de hongos durante un período superior a 150 años (1950 - 2100). A través de esta información, se pretendía aumentar el conocimiento sobre la ecología de los hongos y comprender las interacciones entre el clima y la productividad de los hongos. El estudio se realizó con los datos recopilados de más de 100 parcelas de muestreo, en los bosques de pinos de Cataluña (tomadas las muestras semanalmente durante la fructificación más alta de hongos, entre 1995 y 2016). Los modelos propuestos revelan que las variables climáticas son las más relevantes a la hora de predecir la ocurrencia y la productividad. Las estimaciones anteriores (evolución histórica) de la producción de hongos muestran que no hubo variaciones significativas en el nivel global para Cataluña, mientras que, en general, en el análisis espacialmente explícito del territorio catalán, se detectó una disminución significativa en algunas regiones de los Pirineos. Sin embargo, en los diversos escenarios futuros analizados, no se observaron variaciones significativas en la cantidad de producción comercializada, comestible y total de hongos a nivel global de Cataluña. Mientras tanto, para las especies Hygrophorus latitabundus, Cantharellus lutescens, Hydnum repandum y Lactarius grupo deliciosus (en algunos escenarios), se detectó un aumento generalizado de la producción. Por otro lado, a nivel regional, y de acuerdo con las fluctuaciones climáticas de cada área específica, donde se pudo ver una evolución positiva y negativa significativa de la producción de hongos en Cataluña.

Palabras clave: Hongos, multifuncionalidad, productos forestales no madereros, modelos mixtos.

\begin{abstract}
For this study, there were determined the changes in mushroom production regarding different models and scenarios of climate change and historic records in Catalonia. This data allowed the estimate and prediction of the historical and future evolution of mushroom production for a period longer than 150 years $(1950-2100)$. Through this information, it was intended to growth of knowledge regarding the fungi ecology and understanding the interactions between climate and mushroom productivity. The study was performed by the data gathered from more than 100 sampling plots, in the Catalonia pine forests (taken the specimens weekly during the highest mushrooms fructification, between 1995 and 2016). The proposed models reveal that the climatic variables are the most relevant when it comes to predict the occurrence and productivity. The previous estimations (historical evolution) of mushroom production show that there were no significant variations in the global level for Catalonia, while, generally, in the spatially explicit analysis of the Catalan territory, there were detected a significant decrease for some regions of the Pyrenees. However, in the diverse future scenarios analysed stages, there weren't observed significant variations in the amount of marketed, edible and total mushrooms production related to them at global level. Meanwhile, for the species Hygrophorus latitabundus, Cantharellus lutescens, Hydnum repandum and Lactarius group deliciosus (in some scenarios), it was detected a generalized increasing of the production. Otherwise, it's at regional level, and according to every specifically area's climatic fluctuations, where it could see a significant positive and negative evolutions of mushroom production in Catalonia.
\end{abstract}

Keywords: Fungi, multifunctional, non-wood forest products, mixed models. 


\section{Introducción}

Entender los patrones biogeográficos en la ecología de los hongos y su fructificación es necesario para avanzar en el conocimiento de estos organismos que juegan un papel clave en el funcionamiento de los ecosistemas forestales y en la provisión de numerosos servicios ecosistémicos. En los ecosistemas mediterráneos, en muchas ocasiones caracterizados por una baja rentabilidad para el aprovechamiento de los recursos madereros (Palahí et al., 2009, Alexander et al., 2002), los productos forestales no madereros, entre los que destacan las setas comestibles y comerciales, son de gran importancia desde el punto de vista no solo ecológico, sino también socioeconómico (Voces et al., 2012, Bonet et al., 2013).

Los últimos datos de "Intergovernmental Panel on Climate Change" (IPCC) muestran que los cambios en el clima que vienen observándose en las últimas décadas seguirán en el futuro (Moss et al., 2010). Por ello, se está trabajando con distintos modelos y posibles escenarios de emisiones de gases de efecto invernadero para predecir futuros impactos sobre los ecosistemas terrestres. Teniendo en cuenta la alta relación que guardan variables climáticas como la precipitación o la temperatura con la producción de setas, se estima que dichos cambios en el clima podrían afectar a la producción de estas. Sin embargo, el conocimiento sobre las dinámicas espacio-temporales de la producción micológica en ecosistemas forestales mediterráneos vulnerables frente al cambio climático es muy escaso, haciendo necesario profundizar en la investigación en este campo, para predecir y anticiparse, si es necesario, con una gestión adaptativa de los ecosistemas forestales para mantener la riqueza fúngica (de-Miguel et al., 2014, Collado et al., 2018).

En dicho contexto, este estudio pretende analizar la evolución histórica y futura $(1950$ - 2100) de la producción de setas epigeas en los pinares del noroeste de la Península Ibérica (Cataluña), con especial énfasis en especies de hongos de alto interés comestible y comercial como son Cantharellus lutescens, Hydnum repandum, Hygrophorus latitabundus, Lactarius grupo deliciosus, Macrolepiota procera y Tricholoma terreum. Así mismo, pretende ser pionero en la creación de modelos de producción sensibles al clima para distintas especies para las cuales no existen herramientas predictivas con el objetivo de realizar estimaciones inéditas de la producción histórica y futura considerando múltiples escenarios de cambio climático, a partir del análisis espacio-temporal de una de las series de datos de producción micológica más importantes del mundo.

\section{Material y métodos}

\subsection{Datos}

Para dicho estudio se utilizaron datos de producción de setas de la Universidad de Lleida y el Centro Tecnológico Forestal de Cataluña procedentes de más de 100 parcelas de muestreo permanentes situadas en el territorio catalán, en las que se han 
recogido datos semanalmente durante el principal período de fructificación (agosto-diciembre), entre 1995 y la actualidad. Más información sobre les métodos de muestreo y los datos se pueden encontrar en Bonet et al. 2004 y Martínez de Aragón et al. 2007. Los datos climáticos históricos para toda Cataluña, así como los correspondientes a las parcelas de muestreo micológico, se obtuvieron mediante interpolación y corrección altitudinal (metodología DAYMET, Thornton et al., 1997; Thornton i Running, 1999) utilizando el paquete de R “meteoland” (De Cácereset al. 2017) de las observaciones de las estaciones meteorológicas de la Agencia Estatal de Meteorología y el Servicio Meteorológico de Cataluña, del Proyecto Consolider-Ingenio MONTES (CSD2008-00040). Además, los escenarios futuros de cambio climático se obtuvieron:

1. Del proyecto MONTES, concretamente los escenarios A2 y B2 propuestos por el IPCC-SRES.

1. Del proyecto EU-CORDEX, del cual se utilizaron dos escenarios de emisiones de gases de efecto invernadero RCP4.5 y RCP8.5 combinados con dos modelos climáticos regionales alternativos: RCA4 y CCLM4-8-17.

Por tanto, en total se analizaron seis escenarios alternativos de cambio climático hasta el año 2100, además de las tendencias históricas desde 1950 hasta la actualidad. Finalmente, se utilizaron los datos referentes a las cubiertas vegetales de Cataluña del Mapa de Hábitats CORINE, en su versión actualizada entre los años 2008 y 2012, que utiliza nuevos métodos y tecnología digital LiDAR para obtener información de alta resolución y espacialmente continua sobre los tipos de vegetación.

\subsection{Análisis estadístico}

Para estimar la producción pasada y futura de diferentes especies de setas, tras valorar y probar diversos enfoques alternativos (p. ej. Geographically weighted regression, modelos mixtos o diferentes métodos de interpolación como Kriging), finalmente se optó por la utilización de modelos lineales generalizados mixtos. Estos permiten, a partir de la incorporación de efectos aleatorios, considerar la posible autocorrelación espacio-temporal debida a las características del muestreo de la producción micológica (remedición de parcelas permanentes a lo largo del tiempo y distribuidas por una amplia zona geográfica, de-Miguel et al., 2014; Karavani et al., 2018; Collado et al., 2018). La alta ocurrencia de valores de producción nulos debido al tamaño reducido de las parcelas y la propia estocástica en la aparición de setas, opté por la modelización por partes. Con este método se distingue una primera etapa donde se estima la probabilidad de aparición de setas, a partir de una regresión logística de efectos mixtos, y una segunda etapa donde se predice la producción micológica a partir de los valores no nulos, utilizando modelos lineales (Hamilton i Brickell, 1983; de-Miguel et al., 2014; Karavani et al., 2018; Collado et al., 2018). 
Los modelos ajustados se utilizaron en el análisis espacialmente explícito de la producción micológica anual histórica y futura con una resolución espacial de 1 $\mathrm{km} 2$. Dicho análisis permitió la creación de mapas de producción anual de setas entre 1950 y 2100 en función de los datos meteorológicos históricos y de los diferentes escenarios de cambio climático. A partir de esta información estimé las tendencias tanto desde un punto de vista espacialmente explícito como a escala regional para el conjunto de Cataluña. Para examinar dichas tendencias, se utilizaron métodos no paramétricos como el test de Mann-Kendall (Mann, 1945) y la aproximación de Theil-Sen (Sen, 1968) con el objetivo de determinar la significación y la magnitud de dichas tendencias.

\section{Resultados y discusión}

En lo referente a los modelos de producción micológica, la mayoría de grupos y especies de hongos mostraron una relación significativa y positiva con la precipitación de los meses de agosto a octubre, coincidiendo con el periodo de fructificación de muchas especies. Por otro lado, especies como T. terreum, con una fenología más tardía, presentó una relación significativa y positiva con la precipitación de los meses de septiembre a noviembre. Además, de manera general, se pudo ver que las temperaturas que influyen de manera más significativa en la producción anual de setas son aquellas que resultan más limitantes para la fructificación de estas, principalmente la temperatura máxima media en agosto y la temperatura mínima en noviembre, enmarcando la fructificación de setas en un rango propicio de temperaturas. La producción anual de cada especie concreta presenta ciertas características propias en los modelos, reflejando diferencias ecológicas y fenológicas entre especies.

El hecho que los modelos de producción estén ajustados con un mayor número de variables explicativas respecto a los modelos de probabilidad de aparición, puede deberse a que los primeros abarcan todo el período de fructificación, mientras que los segundos describen las condiciones mínimas necesarias para que las distintas especies analizadas empiecen a fructificar. La elevada significación estadística de la precipitación y las temperaturas del mes de agosto concuerda con la idea de que, en las regiones mediterráneas, las variables más limitantes son las altas temperaturas y la poca precipitación de finales de verano.

Tras analizar las tendencias históricas de la producción de setas, los resultados sugieren que, globalmente para el conjunto de Cataluña, si bien se aprecian tendencias ligeramente negativas, estas no son lo suficientemente significativas como para poder afirmar que ha existido una disminución histórica de la producción micológica ocasionada por cambios en las condiciones climáticas. Por el contrario, a nivel espacialmente explícito sí que se han podido ver disminuciones significativas de la producción en algunas zonas donde la temperatura ha aumentado más notoriamente y la precipitación se ha mantenido más o menos constante desde 1950 hasta la actualidad. A modo ilustrativo de dichas tendencias, se muestra el análisis realizado para la especie Lactarius grupo deliciosus (níscalo) (Fig. 1 y Fig. 2). 


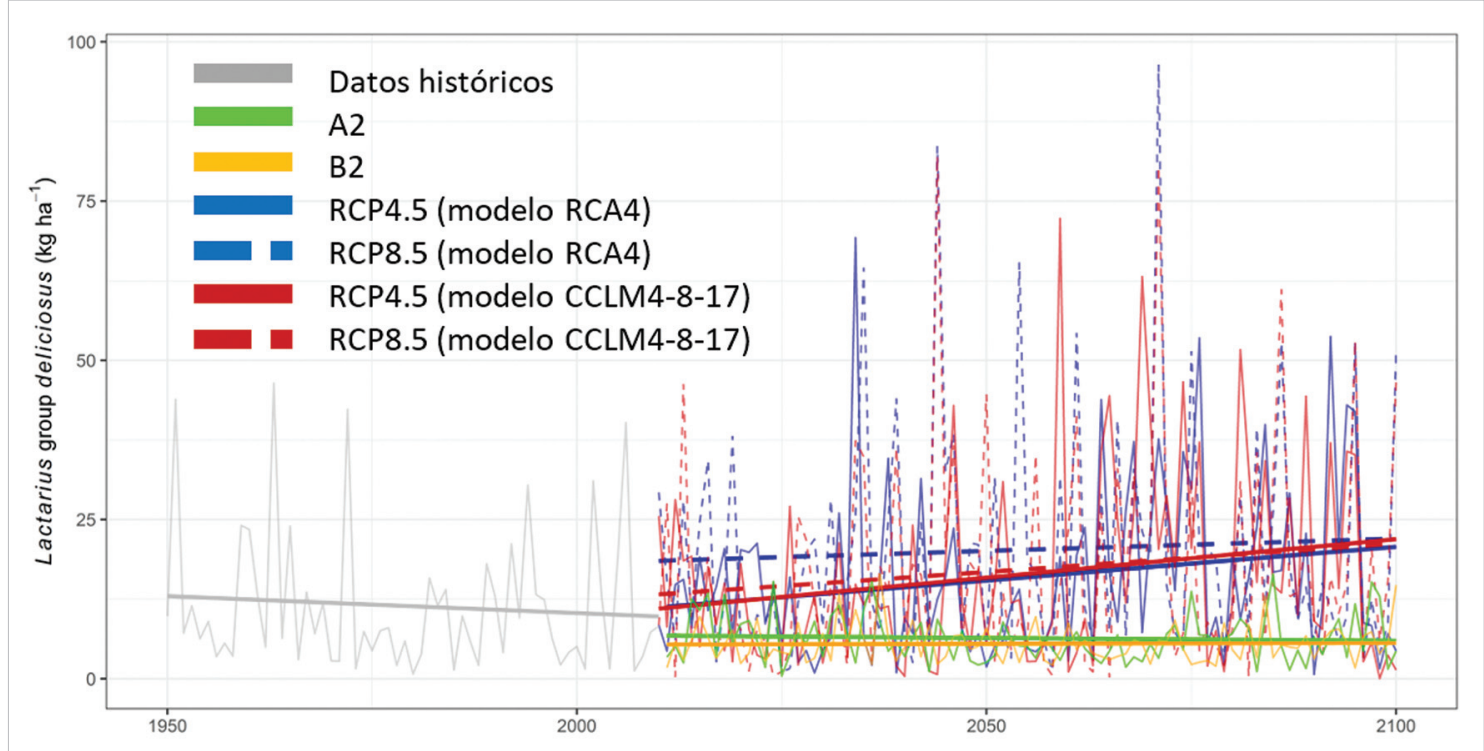

Figura 1. Evolución media de la producción de Lactarius grupo deliciosus para el periodo histórico (1950-2010) y los distintos escenarios y modelos futuros (2011 - 2100), en Cataluña.

De igual manera que en las series históricas, el análisis de los distintos escenarios futuros de cambio climático no arrojó apenas variaciones significativas en la producción micológica a escala regional (para el conjunto de Cataluña). Por el contrario, el análisis espacialmente explícito sí mostró variaciones significativas en la producción micológica futura para determinadas zonas del territorio catalán, siendo estas positivas o negativas en función del resultado de la evolución de las temperaturas y la precipitación en dichas zonas, así como de su interacción. Para los distintos grupos de setas estudiados, las variaciones más significativas ocurrieron en aquellos escenarios en que la temperatura aumentaría de manera más notoria, debido a las mayores emisiones de gases de efecto invernadero (RCP8.5), pero condicionada por la cantidad de precipitación en cada zona. Cabe destacar, también, las diferencias encontradas entre los escenarios propuestos por IPCC-SRES y los resultantes del proyecto EU-CORDEX (p. ej. Fig. 1 para L. grupo deliciosus), que pueden ser considerables para el conjunto de grupos y especies. Por un lado, los escenarios A2 y B2 muestran un rango de datos climáticos más parecidos a los actuales, haciendo que la producción predicha por los modelos sea también algo más coherente con las tendencias históricas. Sin embargo, los datos de precipitación de estos dos escenarios distan mucho de los de los cuatro escenarios basados en EUCORDEX, considerados más actuales y fieles a la realidad.

\section{Futuras líneas de investigación}

En relación a este trabajo y siguiendo la misma línea de estudio, en el futuro se podría plantear la posibilidad de estudiar tanto la diversidad como la fenología de 




Figura 2. Análisis espacialmente explícito de las tendencias futuras en Lactarius grupo deliciosus. En gris se muestra los pinares estudiados donde las tendencias no son significativas, mientras que en una escala de colores se muestra la magnitud de la tendencia según la aproximación de Theil-Sen (en el diagrama de caja se muestra un resumen de los valores del total de pixeles de $1 \mathrm{~km}^{2}$ ). Se distinguen los escenarios RCP4.5 y RCP8.5 del proyecto EU-CORDEX (utilizando los modelos regionales RCA4 y CCLM4-8-17) y A2 y B2 propuestos por IPCC-SRES.

las distintas especies de hongos y si estas guardan alguna relación, como parece evidente, con la productividad de setas. De esta manera se podría obtener información también sobre posibles cambios pasados y futuros no solo en la producción, sino también en la diversidad de hongos. Por otra parte, por falta de datos, en este estudio se ha abordado el estudio de la evolución de la producción micológica en fun- 
ción únicamente del cambio climático sin considerar los cambios históricos y futuros en la estructura de las masas forestales y en el uso del suelo. Por consiguiente, futuros trabajos podrían contribuir a expandir aún más nuestro conocimiento sobre el impacto del cambio global sobre la evolución de la producción de setas teniendo en cuenta factores como la migración de especies forestales arbóreas o los cambios en el área y tipología forestales. Debido a las limitaciones subyacentes a los datos y metodología utilizados se abre la posibilidad de mejorar los resultados de este estudio con nuevas técnicas de análisis (técnicas de análisis de ADN y/o ARN, novedosas técnicas de Machine Learning, etc.), colaboración entre disciplinas y entidades, así como la obtención de más datos que permitan agrandar nuestro conocimiento en este campo.

\section{Bibliografía}

Alexander, S.J.; Pilz, D.; Weber, N.S.; Brown, E.; Rockwell, V.A.; 2002. Mushrooms, trees and money. Value estimates of commercial mushrooms and timber in the Pacific Northwest. Environ. Manage. 30, 129-141. https://doi.org/10.1007/s00267-002-2610-1

Bonet, J.A.; Fischer, C.R.; Colinas, C.; 2004. The relationship between forest age and aspect on the production of sporocarps of ectomycorrhizal fungi in Pinus sylvestris forests of the central Pyrenees. Forest Ecology Management. 203 (1-3), 157-175. https://doi.org/ 10.1016/j.foreco.2004.07.063

Bonet, J.A.; González-Olabarria J.R.; Martínez De Aragón, J.; 2013. Mushroom Production as an Alternative for Rural Development in a Forested Mountainous Area. Journal of Mountain Science. 11(2). https://doi.org/10.1007/s11629-013-2877-0

Collado, E.; Camarero, J.J.; Martínez de Aragón, J.; Pemán, J.; Bonet, J.A.; de-Miguel, S.; 2018. Linking fungal dynamics, tree growth and forest management in a Mediterranean pine ecosystem. Forest Ecology Management. 422, 223-232. https://doi.org/10.1016/ j.foreco.2018.04.025

De Cáceres, M.; Martin-StPaul, N.; Granda, V.; Cabon, A.; 2017. meteoland: Landscape Meteorology Tools. R package version 0.6.4. http://vegmod.ctfc.cat/ meteolandweb.

de-Miguel, S.; Bonet, J.A.; Pukkala, T.; de Aragón, J.M.; 2014. Impact of forest management intensity on landscape-level mushroom productivity: a regional modelbased scenario analysis. Forest Ecology Management. 330, 218-227. https://doi.org/10.1016/j. foreco.2014.07.014

Hamilton, D.A.; Brickell, J.E.; 1983. Modeling methods for a two-state system with continuous responses. Can. J. For. Res. 13, 1117-1121. https://doi.org/10.1139/x83-149

Karavani, A.; De Cáceres, M.; Martínez de Aragón, J.; Bonet, J.A.; de-Miguel, S.; 2018. Effect of climatic and soil moisture conditions on mushroom productivity and related ecosystem services in Mediterranean pine stands facing climate change. Agric. For. Meteorol. 248, 432-440. https://doi.org/10.1016/j.agrformet.2017.10.024

Moran, P.A.P.; 1948. The interpretation of statistical maps. J. R. Stat. Soc. 10, 243-251. https://doi.org/10.1111/j.2517-6161.1948.tb00012.x

Moss. R.H.; Edmonds, J.A.; Hibbard, K.A.; Manning, M.R.; Rose, S.K.; van Vuuren, D.P.; Carter, T.R.; Emori, S.; Kainuma, M.; Kram, T.; Meehl, G.A.; Mitchell, J.F.B.; Nakice- 
novic, N.; Riahi, K.; Smith, S.J.; Stouffer, R.J.; Thomson, A.M.; Weyant, J.P.; Wilbanks, T.J.; 2010 The next generation of scenarios for climate change research and assessment. Nature 463, 747-756. https://doi.org/10.1038/nature08823

Palahí, M., Pukkala, T., Bonet, J.A., Colinas, C., Fischer, C.R., Martínez de Aragón, J., 2009. Effect of the inclusion of mushroom values on the optimal management of evenaged pine stands of Catalonia. For. Sci. 55 (6), 503-511.

Sen, P.; 1968. Estimates of the regression coefficient based on Kendall's tau. J. Am. Stat. Assoc. 63, 1379-1389. https://doi.org/10.1080/01621459.1968.10480934

Thornton, P.E.; Running, S.W.; 1999. An improved algorithm for estimating incident daily solar radiation from measurements of temperature, humidity, and precipitation. Agric. For. Meteorol. 93, 211-228. https://doi.org/10.1016/S0168-1923(98)00126-9

Thornton, P.E.; Hasenauer, H.; White, M.A.; 2000. Simultaneous estimation of daily solar radiation and humidity from observed temperature and precipitation: an application over complex terrain in Austria. Agric. For. Meteorol. 104, 255-271. https://doi.org/ 10.1016/S0168-1923(00)00170-2

Voces, R.; Díaz-Balteiro, L.; Alfranca, O.; 2012. Demand for wild edible mushrooms. The case of Lactarius deliciosus in Barcelona (Spain). J. For. Econ. 18 (1), 47-60. https:// doi.org/10.1016/j.jfe.2011.06.003 
